Paper No. 6350

\title{
The relation between the yield of a discharging well at equilibrium and its diameter, with particular reference to a Chalk well $\dagger$
}

\author{
by
}

Jack Ineson, B.Sc., Ph.D.

\section{Discussion}

Professor N. S. Boulton (Professor of Civil Engineering, University of Sheffield) observed that the Author had shown convincingly by statistical analysis of extensive field data that the diameter of a well in a fissured aquifer had much more influence on the discharge than the Dupuit equation indicated. To explain this influence he considered certain models comprising simple fissure patterns, though in $\$ 34$ he rightly suggested that further knowledge of the actual fissure distribution was needed before a fully satisfactory explanation of the observed yield-diameter relations could be given. The Author's hypothetical models were valuable in showing possible effects of fissures, but their limitations should be remembered.

53. Referring to the grid of two sets of parallel fissures ( $\$ 36$ and Table 8 ), the Author had apparently assumed that the two sets were not hydraulically connected at points where they intersected. It seemed probable, however, that some intersections would be connected and it was useful to consider a simple model in which the fissures were connected at all intersections. For example, considering a well-axis located at the intersection of two fissures of a set of fissures forming squares, having a length of side $r$, Professor Boulton had calculated the relative yield by relaxation methods for well diameters of $r$ and $2 r$. For this calculation it had been assumed that the extent of the cone of depression was $R=250$, and that Darcy's law applied to the flow in the fissures. The result showed that doubling the diameter of the well increased the yield by only $18 \%$. For comparison, the corresponding increase in yield for non-connected fissures (putting $n=2$ in Table 8 , line 3 ) was $200 \%$ and, according to the Dupuit equation for a homogeneous aquifer, $13 \%$. This rather artificial example showed the importance of hydraulic continuity at fissure intersections. For a more realistic approach a model comprising a probable distribution of the intersection points should be considered.

54. The effect of a very small lateral seepage between adjacent fissures was shown by considering a simple model which might be relevant for some chalk aquifers. Assume a two-dimensional system of small parallel fissures so numerous that the permeability $k_{y}$ in the direction of the fissures might be taken as uniform. A much smaller permeability $k_{x}$ at right-angles to the fissures was also assumed. The rate of discharge for a fixed drawdown might then be found by the well-known procedure of considering the transformed aquifer having isotropic permeability. The resulting flow-system in which the stream lines were hyperbolas and the "equipotential" lines ellipses had been described by Muskat 8 . For example, if $k_{y} / k_{x}=100$, and if the extent of the cone of depression $R=250 \mathrm{ft}$ in the direction of the fissures, it was found that the discharge for a well $4 \mathrm{ft}$ diameter was only $22 \%$ greater than for a well $2 \mathrm{ft}$ diameter. The increase

† Proc. Instn civ. Engrs, vol. 13, p. 299 (July 1959). 
in discharge with diameter was therefore greatly reduced by the small lateral permeability between fissures.

The Author, in reply, said that he was grateful to Professor Boulton for emphasizing the limitations in the use of the hypothetical model for fissure flow into a well. The adopted model related to two sets of fissures which were not in hydraulic continuity throughout the entire system, giving rise to an extreme case of inflow into a well through fissures. On the other hand, it could equally well be argued that no increase in yield would occur with increase in diameter of a well, when all fissures of the hypothetical model were in complete hydraulic continuity from the outer to the inner boundaries of the flow system. The limiting case had been used to emphasize an extreme example of fissure flow into a well. The distribution of fissures and the degree of their hydraulic continuity would lead to a complete range of results between the two limiting cases of the hypothetical models dependent upon the hydrogeological conditions obtaining in an aquifer, as repeatedly demonstrated underground during examination of fissure flow into Chalk wells. In the same way, the degree of contribution from the main mass of the aquifer into the fissure system would vary considerably.

56. The calculation of lateral seepage into a two-dimensional system of parallel fissures was particularly interesting. From the statistical analyses of multiple correlation (equation (8)) for Chalk wells, the increase in yield of a discharging well with increase in diameter from $2 \mathrm{ft}$ to $4 \mathrm{ft}$ could be shown by extrapolation to range from 23 to $28 \%$, according to the type of ground-water flow conditions in the aquifer. These values would compare favourably with the theoretical calculations of Professor Boulton.

\section{REFERENCE}

8. M. Muskat, "Flow of homogeneous fluids". McGraw-Hill (1937), p. 184. 